\title{
Analysis of Putative Amino Acid Signals Within Human T Cell Leukemia Virus Type I (HTLV-I) Tax Protein Involved in Mediating Tax Secretion
} Sharron L Manuel ${ }^{* \ddagger}$, Pooja Jain, Katie Flaig and Brian Wigdahl

Address: Department of Microbiology and Immunology, and Institute for Molecular Medicine and Infectious Disease, Drexel University College of Medicine, Philadelphia, PA, USA

* Corresponding author $\ddagger$ Presenting author

from 2005 International Meeting of The Institute of Human Virology

Baltimore, USA, 29 August - 2 September 2005

Published: 8 December 2005

Retrovirology 2005, 2(Suppl I):P63 doi:10.1 I86/I742-4690-2-SI-P63

HTLV-1 oncoprotein Tax protein is known to be released from HTLV-1-transformed cells by a mechanism other than cell death; however, the mechanism of Tax secretion remains to be established. This study elucidates domains within Tax that contribute to its subcellular localization and secretion. Analysis of the amino acid sequence of Tax has revealed the presence of four putative secretory signals within the carboxy-terminal domain namely YTNI, LL, DHE and terminal V. Mutation of two putative signals (YTNI and DHE) resulted in aberrant subcellular localization of Tax, with cytoplasmic Tax accumulating in structures corresponding to the ER and Golgi. We have also studied the effect of these mutations on the secretion of Tax in baby hamster kidney cell (BHK-21) line. The results have demonstrated that mutating YTNI to ATNI resulted in approximately a 2 -fold reduction in secretion. Mutation of LL to AA abrogated the intake of Tax, therefore, no secretion was observed. Mutating DHE and terminal V did not show any effect on Tax secretion, however, a combination of DHE mutation with YTNI mutation or AA mutation resulted in an altered intake and secretion of Tax. These studies substantiate a link between cytoplasmic Tax and its subsequent secretion and also indicate potential amino acid signals that might direct secretion of Tax. 